\title{
Evaluation of pilot implementation of seasonal malaria chemoprevention on morbidity in young children in Northern Sahelian Ghana
}

Patrick O. Ansah', Nana A. Ansah' ${ }^{1}$, Keziah Malm², Dennis Awuni ${ }^{1}$, Nana Peprah², Sylvester Dassah', Sobe Yarig ${ }^{1}$, Charles Manful ${ }^{1}$, John Agbenyeri ${ }^{4}$, John Awoonor-Williams ${ }^{3}$, Wilfred Ofosu ${ }^{5}$ and Abraham R. Oduro ${ }^{1 *}$ (e)

\begin{abstract}
Background: In Sahelian Africa, the risk of malaria increases with the arrival of the rains, particularly in young children. Following successful trials, the World Health Organization (WHO) recommended the use of seasonal malaria chemoprevention (SMC) in areas with seasonal peak in malaria cases. This study evaluated the pilot implementation of SMC in Northern Ghana.

Methods: Fourteen communities each serving as clusters were selected randomly from Lawra District of Upper West Region as intervention area and West Mamprusi District in the Northern Region as the non-intervention area. The intervention was undertaken by the National Malaria Control Programme in collaboration with regional health directorates using sulfadoxine-pyrimethamine plus amodiaquine and standard WHO protocols. Before and after surveys for malaria parasitaemia and haemoglobin levels as well as monitoring for malaria morbidity and mortality were undertaken.
\end{abstract}

Results: At the end of the intervention, participant retention was 92.9\% (697/731) and 89.5\% (634/708) in the intervention and the non-intervention areas, respectively. The proportion of children with asexual parasites reduced by $19 \%(p=0.000)$ in the intervention and increased by $12 \%(p=0.000)$ in the non-intervention area. Incidence rates of severe malaria were 10 and 20 per 1000 person-years follow up in the intervention and comparison areas, respectively with P.E of $45 \%(p=0.62)$. For mild malaria, it was 220 and 170 per 1000 person-years in intervention and comparison area, respectively with PE of - $25 \%(p=0.31)$. The proportion of children with anaemia defined as $\mathrm{Hb}<11.0 \mathrm{~g} /$ $\mathrm{dl}$ reduced from $14.2 \%$ (52.8-38.6\%) in the intervention area as compared to an increase of $8.1 \%$ (54.5\% to 62.6) the non-intervention arm, Mean Hb reduced by $0.24 \mathrm{~g} / \mathrm{dl}(\mathrm{p}=0.000)$ in the non-intervention area and increased of $0.39 \mathrm{~g} /$ $d l(p=000)$ in the intervention area.

Conclusions: The feasibility and effectiveness of SMC introduction in Northern Ghana was demonstrated as evidenced by high study retention, reduction in malaria parasitaemia and anaemia during the wet season.

Keywords: Feasibility, Seasonal-malaria-chemoprevention, Children, Northern Ghana

*Correspondence: aroduro@gmail.com

${ }^{1}$ Navrongo Health Research Centre, Research and Development Division, Ghana Health Service, Navrongo, Ghana

Full list of author information is available at the end of the article

\section{Background}

Malaria remains a major public health concern in SubSaharan Africa (SSA) despite a significant reduction in the global burden of the disease in the last two decades 
[1]. Sub-Saharan Africa still accounts for most clinical cases and malaria-related deaths, which contributes about $10 \%$ of all under-five deaths. Malaria exerts negative social and economic impact in endemic areas. Economically it reduces productivity due to worker absenteeism and increased national health care spending. Socially, malaria affects individual and household behaviours resulting in broad social costs with the attendant long-term effect on growth and development $[1,2]$.

Currently, the World Health Organization (WHO) recommends a combination of approaches for malaria control including the use of long-lasting insecticide-treated nets (ITNs), indoor residual spraying (IRS), confirmation and use of artemisinin-based combination treatment (ACT) for case management $[1,2]$. In addition, intermittent preventive treatment in pregnancy (IPTp) and children (IPTc) are recommended for specific highrisk groups $[1,2]$. The IPTc has been found to be a safe method of malaria control that reduces significant proportion of clinical malaria illness in areas of marked seasonal malaria transmission. It also has substantial protective effect against all-cause mortality. Therefore, IPTc as valuable tool can contribute to the control of malaria especially in children in areas with short and marked seasonal transmission [3].

The WHO currently recommends Seasonal Malaria Chemoprevention (SMC) as a tool to prevent malaria among young children in areas with high seasonal transmission especially in the Sahelian areas of Africa [4, 5]. The SMC is the intermittent administration of full malaria treatment course to children during the malaria season. This is to check infection by sustaining therapeutic concentrations of anti-malarial drugs in the blood during the period of highest malarial risk $[4,5]$. The WHO recommends sulfadoxine-pyrimethamine plus amodiaquine as the drugs of choice in the Sahel sub-region of Africa, where Plasmodium falciparum is sensitive to both anti-malarial drugs $[4,5]$. Across the Sahel sub-region, most childhood malarial disease and deaths occur during the rainy season, which is generally short (3-4months). Giving effective anti-malarial treatment at monthly intervals during this period protects against uncomplicated and severe malaria in young children [6-8].

The SMC approach is cost-effective and safe, and can be administered by community-health workers. In areas where SMC has been adopted it has helped in reducing the cases of malaria and proven to be very effective [6-8]. Apart from reducing infection, morbidity and mortality, malaria anaemia and child wellbeing are also improved [6-8]. Several studies in different countries attest that SMC is very effective in the fight against malaria and should be scaled up in other areas that had more cases of malaria [6-8].
Despite the success, the SMC strategy has some challenges. The implementation and acceptance of treatment drugs have been an issue for some time. The issue of implementation particularly the last mile distribution across hard-to-reach areas have often emerged. Also, studies have shown that some mothers do not administer the anti-malarial medication as prescribed. Parents give several reasons for not adhering to the medication, such as the drugs being bitter and complaints of the negative side effects they get from these drugs. Despite this, there is good adherence to the SMC drugs in most areas where it has been implemented, and care givers have attested to them being beneficial by helping them in reducing the incident cases of malaria $[9,10]$.

The Ghana National Malaria Control Programme (NMCP) in 2014 proposed to adopt SMC using the sulfadoxine-pyrimethamine plus amodiaquine combination in Ghana [11]. The adoption was to help reduce morbidity and mortality in children in the northern Ghana where malaria transmission is seasonal. This was to also improve the wellbeing and the livelihoods of many children and parents as time and resources are spent to cater for malaria that could be channeled into other productive ventures. However, there was a need for the NMCP to undertake a pilot study to determine the feasibility and effectiveness of SMC in the Ghanaian setting to inform policy decision for its scale-up.

\section{Methods \\ Study setting}

The evaluation was undertaken by the Navrongo Health Research Centre (NHRC), a field station of the Research and Development Division of the Ghana Health Service [12]. The NHRC runs the Navrongo Health and Demographic Surveillance System (NHDSS), a platform designed to provide efficiency in evaluating health and social interventions [12]. The intervention and the nonintervention took place in two different administrative districts; Lawra district, Upper West Region and West Mamprusi in Northern Region both located in the northern part of Ghana [12-14]. Figure 1 shows the location of the NHRC and the two administrative districts in the respective regions. The study setting is characterized by dryness due to its proximity to the Sahel and Sahara. The vegetation is predominantly Savannah grasslands with clusters of highly drought-resistant trees. There are two main seasons in the area, the dry and wet seasons. The dry season stretches from November to March and the wet season from April to October with a peak in July to September which coincides with malaria transmission. Malaria in northern Ghana is very seasonal [15-17]. Figures 2 and 3 provides historical data from the outpatient departments of the health facilities in the administrative 


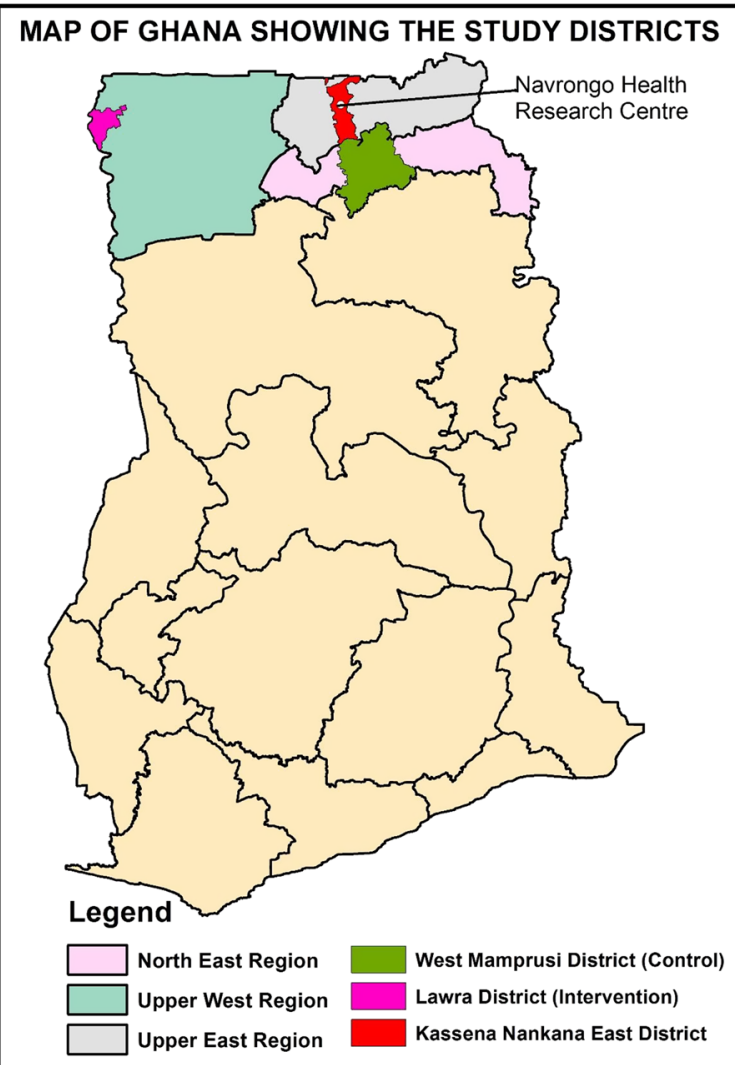

Fig. 1 Map of Ghana showing the regions and districts of the intervention and control areas and that of the Navrongo Health Research centre

regions that demonstrate seasonal malaria incidence in the two study districts.

In northern Ghana cases of malaria are seasonal caused mainly by $P$. falciparum and transmitted by anopheles' mosquitoes with Anopheles gambiae sensu lato (s.l.) and Anopheles funestus being the predominant vector species [15-17].

\section{Study areas}

The study districts have similar seasonal characteristics of malaria cases (Figs. 2 and 3) making them well suited for this study. The intervention area was the Lawra district [13] which lies in the North-Western corner of the Upper West Region of Ghana between latitudes $2^{0} 25^{\prime \prime} \mathrm{W}$ and $2^{\circ} 45^{\prime \prime} \mathrm{W}$ and longitudes $10^{\circ} 20^{\prime \prime} \mathrm{N}$ and $11^{\circ} 00^{\prime \prime} \mathrm{N}$. The total population of the area was about 100,929 people. The district had five health centres, ten Communitybased Health Planning and Services (CHPS) compounds [18], three pharmacy shops, one clinic and one hospital which provides health services to the people in the area.

The non-intervention area was at the West Mamprusi District [14], one of twenty districts in the Northern
Region of Ghana and lies within longitudes $0^{\circ} 35^{\prime} \mathrm{W}$ and $1^{\circ} 45^{\prime} \mathrm{W}$ and latitudes $9^{\circ} 55^{\prime} \mathrm{N}$ and $10^{\circ} 35^{\prime} \mathrm{N}$. They had a total population of about 180,877 . There was one polyclinic, one health centre, three private clinics, 6 CHPs compounds, and 15 pharmacy shops in this area.

\section{Study design}

The study used a quasi-experimental design with before and after cross-sectional surveys. The implementation was during the main malaria transmission season from July 2015 to the end of the study in December 2015. The duration of participation for each child was six months. Enrolment was conducted over 2 weeks period and the post-implementation evaluation surveys lasted for 2 weeks. The study population included children aged 3 months to 59 months who were eligible for enrolment in the selected households and communities. Two crosssectional surveys, one at baseline and the other at end line after 6 months were used for evaluation.

The baseline survey took place from July 15, 2015 till July 27, 2015 and first dose was administered in July 27, 2015 and administered for four conservative months at monthly intervals. Continuous monitoring took place from July 27 till December 17, 2015. End line survey was done from December 10, 2015 till December 17, 2015. The enrolled children were followed up between the two surveys for episodes of severe and mild malaria. Other parameters documented included weight, height, malaria parasitaemia, haemoglobin level and temperature.

\section{Sample size estimation}

The sample size calculation was based on the impact of SMC on severe malaria as a primary outcome. Effectiveness assumption is based on studies that showed a reduction in severe malaria by 65 to $86 \%$ [6-9]. Using an average proportion of 0.36 of children under 5 years developing severe malaria and 50 children per cluster with an inter-cluster variation of 0.26 , approximately 14 clusters were needed per arm to demonstrate a $50 \%$ reduction in the proportion of under-five children developing severe malaria at a power of $90 \%$ and a two-sided significance level of $5 \%$. A cluster was taken as the community of residence as outlined by the Ghana Health Service District Health Directorate. Each community was a well-delineated area with defined boundaries.

\section{Sampling strategy}

All districts in the Upper West region were listed and one district (Lawra) was selected at random for evaluation of SMC. The control district, West Mamprusi, was selected at random from the Northern Region which had the same routine interventions against malaria as the Upper West Region apart from SMC. All communities in 


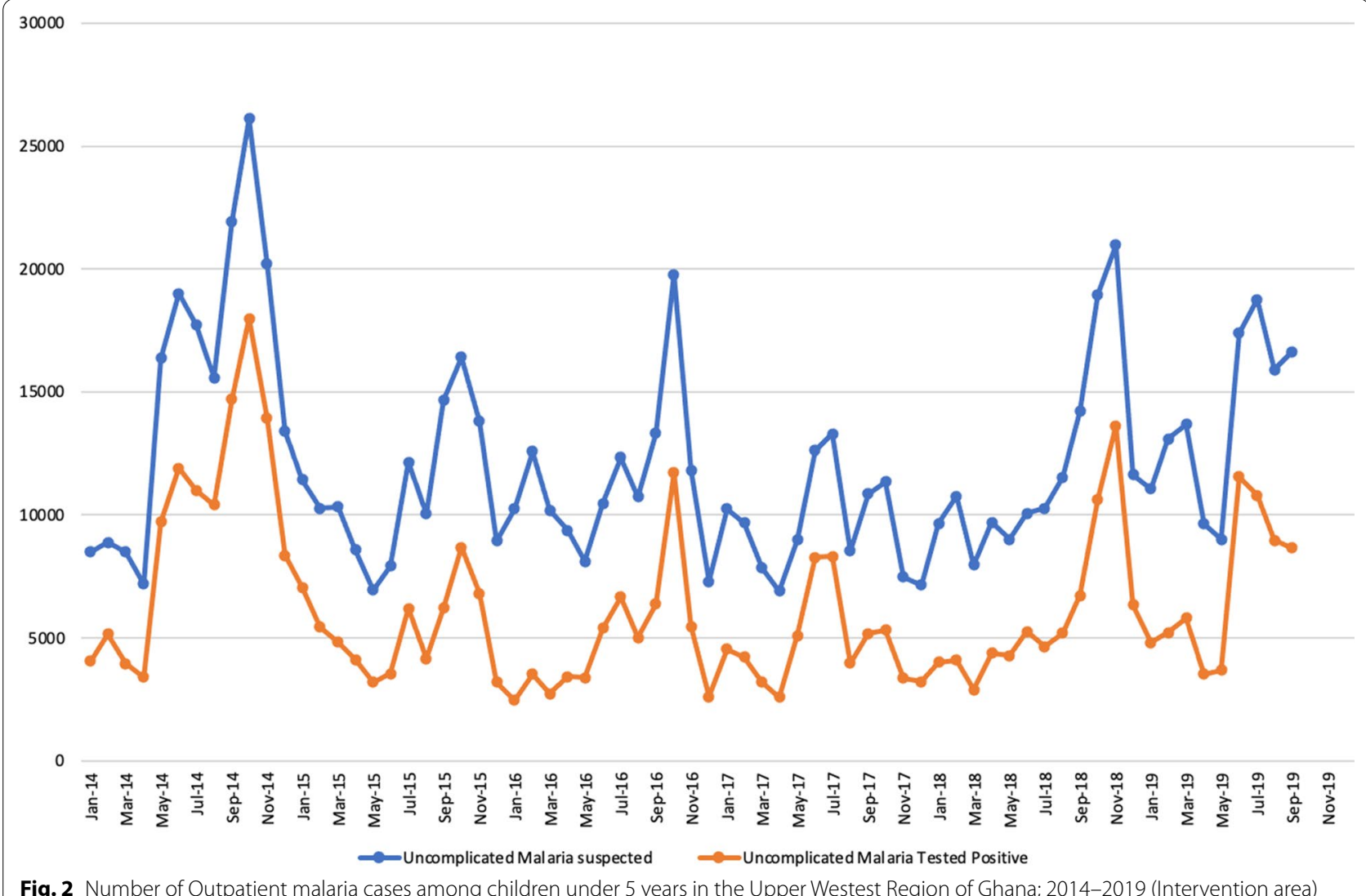

Fig. 2 Number of Outpatient malaria cases among children under 5 years in the Upper Westest Region of Ghana; 2014-2019 (Intervention area)

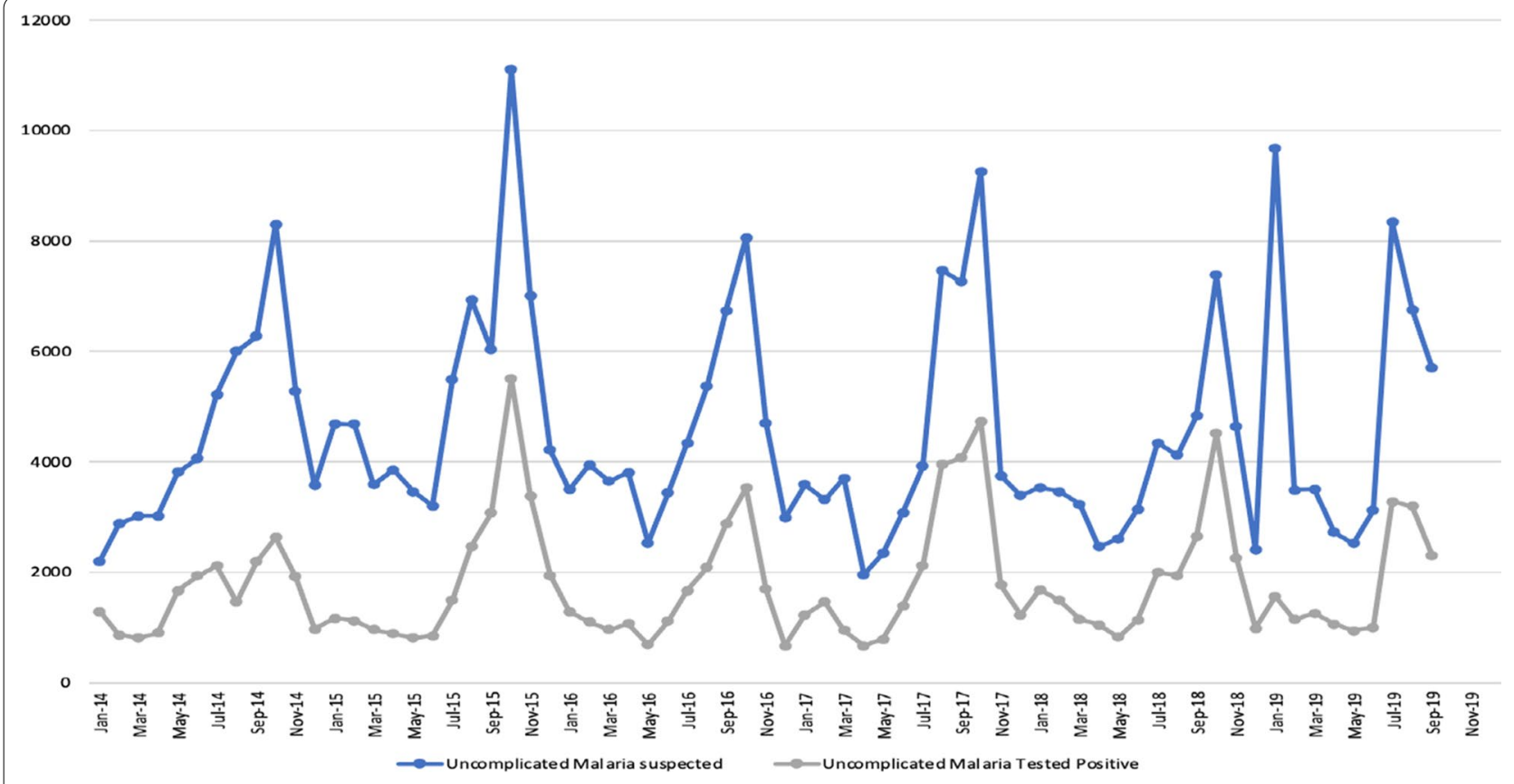

Fig. 3 Number of Outpatient malaria cases among children under 5 years in the North East Region of Ghana; 2014-2019 (Non-intervention area) 
the selected districts were subsequently listed and fourteen out of 141 and 152 communities from Lawra district and West Mamprusi districts respectively were randomly selected. Each selected community was defined as the cluster, the unit of analysis. All the households in each selected community were labelled and listed. All households with eligible children were listed and 40 households randomly selected. In each selected household all eligible children were selected for participation and out of these the children whose guardians gave parental consent and were available for the 4-month duration of the study were selected for inclusion.

A minimum of 50 children per village and 700 children per arm were recruited into the study. Where the community was too small to provide a minimum of 50 participants, the nearest community was added to it to make up a bigger community. Staff visited each household to explain the study in the local language, provided an information sheet, and sought signed consent from parents. Children whose parents consented were enrolled, and mothers/caregivers were issued with a unique identification card bearing the details and study number for each eligible child in their care. The cards were used to identify children if malaria was diagnosed and, for those in clusters randomized to receive SMC, to document SMC courses of treatment received.

\section{Study drug administration}

Study drugs were supplied by National Malaria Control Programme for the regional health directorate. Formulations of sulfadoxine-pyrimethamine $500 / 25 \mathrm{mg}$ tablet (SP) and amodiaquine (AQ) $153 \mathrm{mg}$ tablet were used. Infants 3- 11 months old were dosed with half of a $153 \mathrm{mg}$ tablet of amodiaquine given once daily for three consecutive days and a single dose of half of $500 / 25 \mathrm{mg}$ tablet of SP. Children 12-59 months were given a full tablet of $153 \mathrm{mg}$ amodiaquine base given once daily for three consecutive days and a single dose of a full tablet of $500 / 25 \mathrm{mg}$ tablet of SP. The administration was done by direct observation therapy on the first day and given by community health volunteers. The caregiver gives the rest of the medication on second and third days. This was the intended means of administration by the NMCP. Children received medication for four consecutive months at monthly intervals between July and November 2015.

To determine the proportion of children who experienced an adverse event, study participants in the intervention district were visited at home daily for three days during the administration of SMC and two days after the last dose in each round of drug administration and a side effects questionnaire was completed to document and quantify adverse events that might have occurred since receiving the trial medication. Grading of the severity of adverse events was done by trained field workers. An adverse reaction was graded as mild (grade 1) if it was easily tolerated and moderate to severe (grade 2) if it interfered with normal activity or required treatment.

\section{Malaria surveillance}

A passive surveillance system to monitor malaria episodes was set up at the district hospitals and health centers in the study areas. Fieldworkers were stationed at health facilities and in the intervention and nonintervention districts to pick up any episodes of mild or severe malaria and checked for malaria parasitaemia before treatment was given. Field workers visited the children once a week during the period of drug administration to enquire about their health and completed a morbidity form if a child had any illness. If a child had a history of fever or vomiting within the past $48 \mathrm{~h}$ the parents were advised to take their child to the nearest health facility for examination and treatment. Any mortality that occurred outside the health facility was to be investigated and a verbal autopsy questionnaire administered to help ascertain the cause of death at the Navrongo Health Research Centre [12].

All children with fever were encouraged to attend the health post or health centre to be tested with an rapid diagnostic test (RDT) and were treated with artemetherlumefantrine if tested positive. Consultations were recorded in a health facility register to document the test results and treatment given. In the intervention area, if a child was unwell on the day of the SMC visit, caregivers were asked to bring the child to the health post or hospital for testing with malaria RDT and treatment with artemether-lumefantrine if positive. Those who subsequently tested negative received SMC and were referred to the nearest hospital.

Treatment of study participants seen at the health facilities for other conditions was carried out per national guidelines. Registers for recording SMC administration with a list of all children enrolled in each village were made available for each field worker. In SMC villages, the dose of SP and AQ administered to each child was recorded in a register. All consultations for illness were also recorded in the registers and other details including date, symptoms, RDT results and treatment in case report forms. Field workers were recruited and trained to prepare thick and thin films for microscopic examination to crosscheck the RDT results.

\section{Laboratory methods}

Capillary blood was used to prepare two malaria slides for diagnosis. The slides were stained using a 10\% Giemsa solution and one examined using x100 oil immersion microscopic lens and the other archived for confirmatory 
testing. A sample was considered negative only after 200 high power fields had been read. Parasite counts were converted to parasites per microliter $(\mu \mathrm{l})$, assuming a white blood cell count of 8000 leukocytes per $\mu$ lof blood. In instances where there were discrepancies in the findings in a slide between the two initial technicians (positive or negative or a $50 \%$ or more difference in parasite density), a senior microscopist read the slide and his reading was deemed to be the correct reading. Haemoglobin was measured using Hemocue ${ }^{\circledR}$ (Hb 801 system).

\section{Data management and statistical analysis}

All consultations for illness were recorded and the register checked for completeness during weekly supervision visits. Completed forms by trained personnel on the study were checked by field supervisors and data managers for consistency and accuracy before logging it out for data entry. All data collected were entered twice into a database using EpiData software. Again, automatic checks for consistency and range errors were done, and queries resolved before the dataset was locked for analysis. Effects of SMC on the prevalence of parasitaemia, gametocyte carriage, mean haemoglobin concentration, and proportions of mild anaemia defined as $\mathrm{Hb}<110 \mathrm{~g} / \mathrm{l}$ ) and severe anaemia defined as $\mathrm{Hb}<60 \mathrm{~g} / \mathrm{l}$ ) were estimated from the survey at the end of the transmission season. The results are in descriptive and analytic statistics and also presented in tables and figures. Analyses were performed using Stata version 14 (Stata Corp, College Station, Texas).

\section{Results}

Baseline characteristics: Seven hundred and thirty-one (731) children were recruited from the intervention district and 708 from the control district. At the end of the intervention, participant retention was $92.9 \%$ (697/731) and $89.5 \%(634 / 708)$ in the intervention and the nonintervention areas respectively. Table 1 shows the background characteristics of the children recruited in both districts. Overall, $54.1 \%$ of participants were males and most of the children were aged 25 - 59 months (60.1\%). The average weight of children at baseline in control district was $11.5 \mathrm{~kg}(95 \% \mathrm{CI} 11.09,11.90)$ and in the intervention district was $11.09 \mathrm{~kg}$ ( (95\%CI 10.87, 11.32). Again, the average height (in metres) among children at baseline in the control district was $82.71(95 \% \mathrm{CI} 81.82,83.61)$ and $86.93(95 \% \mathrm{CI} 86.05,87.81)$ in the intervention district.

Overall reported bed net use was almost universal in intervention district (99.5\%) as against $69.5 \%$ in the non-intervention district. Though indoor residual spraying against malaria was being used by both districts, 57 (8.1\%) of the participants in the non-intervention district have not had their house sprayed. For children reporting fever in the week before the survey, 27 (3.8\%) of children

Table 1 Background socio-demographic characteristics of the study participants

\begin{tabular}{|c|c|c|c|c|c|}
\hline \multirow[t]{2}{*}{ Attributes } & \multirow[t]{2}{*}{ Category } & \multicolumn{2}{|c|}{ Control district $(\mathrm{N}=708)$} & \multicolumn{2}{|c|}{ Intervention district $(\mathrm{N}=731)$} \\
\hline & & n (\%) & $(95 \% \mathrm{Cl})$ & n (\%) & $(95 \% \mathrm{Cl})$ \\
\hline \multirow[t]{2}{*}{ Sex } & Male & $385(0.54)$ & $(0.49,0.59$ & $393(0.54)$ & $0.49,0.59$ \\
\hline & Female & $323(0.46)$ & $0.41,0.51$ & $338(0.46)$ & $0.41,0.51$ \\
\hline \multirow[t]{4}{*}{ Birth order } & First & $156(0.22)$ & $0.15,0.29$ & $202(27.6)$ & $0.24,0.36$ \\
\hline & Second & $204(0.28)$ & $0.22,0.34$ & $181(24.8)$ & $0.19,0.31$ \\
\hline & Third & $146(0.21)$ & $0.14,0.28$ & $144(19.7)$ & $0.13,0.27$ \\
\hline & Four or more & $202(0.30)$ & $0.24,0.36$ & $204(0.28)$ & $0.22,0.34$ \\
\hline \multirow[t]{4}{*}{ Age groups } & $3-6$ months & $61(0.09)$ & $0.07,0.11$ & $38(0.05)$ & $0.04,0.07$ \\
\hline & $6-12$ months & $106(0.15)$ & $0.12,0.18$ & $60(0.08)$ & $0.06,0.10$ \\
\hline & 13-24 months & $154(0.22)$ & $0.19,0.25$ & $155(0.20)$ & $0.17,0.23$ \\
\hline & 25-59 months & $387(0.55)$ & $0.51,0.59$ & $478(0.65)$ & $0.61,0.68$ \\
\hline \multirow[t]{4}{*}{ Educational status of care giver } & None & $649(0.92)$ & $0.90,0.94$ & $478(0.65)$ & $0.61,0.68$ \\
\hline & Primary & $34(0.05)$ & $0.03,0.07$ & $196(0.27)$ & $0.24,0.30$ \\
\hline & Secondary & $17(0.02)$ & $0.01,0.03$ & $39(0.05)$ & $0.04,0.07$ \\
\hline & Tertiary & $8(0.01)$ & 0.01 .0 .02 & $18(0.02)$ & $0.01,0.03$ \\
\hline Temp. $\geq 37.5^{\circ} \mathrm{C}$ & Yes & $10(0.014)$ & $0.004,0.020$ & $30(0.04)$ & $0.03,0.06$ \\
\hline Anti-malarial use in $\leq 2$ weeks & Yes & $3(0.004)$ & $0.009,0.012$ & $26(0.04)$ & $0.03,0.06$ \\
\hline IRS & Yes & $651(0.92)$ & $0.90,0.94$ & $720(0.98)$ & $0.97,0.99$ \\
\hline ITN Use & Yes & $493(0.70)$ & $0.67,0.73$ & $727(0.99)$ & $0.98,1.00$ \\
\hline Fever $\leq 2$ weeks & Yes & $27(0.04)$ & $0.03,0.06$ & $40(0.06)$ & $0.04,0.08$ \\
\hline
\end{tabular}


in non-intervention district reported having fever compared with $40(5.5 \%) \%$ of children in the intervention district. However, 30 (4.1\%) of children in intervention district compared with $10(1.1 \%)$ in the non-intervention district had temperature $\geq 37.5^{\circ} \mathrm{C}$ at the time of the enrolment. The use of anti-malarial two weeks before the survey was higher 26 (3.6\%) in intervention district compared with $3(0.4 \%)$ in non-intervention district (Table 1$)$.

Incidence rates of confirmed severe and uncomplicated malaria: A total of nine (9) cases of severe malaria were recorded in the two districts. There were three (3) cases in the intervention area and six (6) in the nonintervention area including one (1) death. The incidence rates of severe disease in the intervention district were 10 per 1000 person-years at risk as against 20 per 1000 person-years at risk in the non-intervention district with an adjusted rate ratio of intervention to non-intervention district of 0.52 giving protective effectiveness of $48 \%$ $(\mathrm{p}=0.62)$ against malaria (Table 2$)$. For clinically diagnosed malaria with any level of parasitaemia, the incidence rates were 220 and 170 per 1000 person-years at risk for intervention and non-intervention areas, respectively (Table 2). There was only one death in the study due to severe malaria and it occurred in the non-intervention district.

Effect of SMC on parasitaemia (Table 3): The proportion of children with parasites increased significantly from $9.0 \%(95 \%$ CI 7,11$)$ at baseline to $21.5 \%$ (95\% CI $18,24)$ at the end-line in the non-intervention district $(\mathrm{P}<0.0001)$. In the intervention district however, the

Table 2 Impact of seasonal malaria chemoprevention on clinical malaria

\begin{tabular}{|c|c|c|c|c|c|c|c|c|}
\hline \multirow[t]{2}{*}{ Outcomes } & \multicolumn{2}{|c|}{ Intervention area } & \multicolumn{2}{|c|}{ Nonintervention area } & \multirow[t]{2}{*}{ Unadjusted IRR } & \multirow[t]{2}{*}{ Adjusted IRR } & \multirow[t]{2}{*}{$\mathrm{PE}(95 \% \mathrm{Cl})$} & \multirow[t]{2}{*}{ P-value } \\
\hline & $\begin{array}{l}\text { n episodes } \\
\text { (years at } \\
\text { risk) }\end{array}$ & $\begin{array}{l}\text { Incidence rate } \\
(95 \% \mathrm{Cl})\end{array}$ & $\begin{array}{l}\text { n } \\
\text { episodesyears } \\
\text { at risk }\end{array}$ & $\begin{array}{l}\text { Incidence rate } \\
(95 \% \mathrm{Cl})\end{array}$ & & & & \\
\hline \begin{tabular}{l}
\multicolumn{1}{c}{ Malaria } \\
diagnosis with \\
any asexual para- \\
sitaemia
\end{tabular} & $55(241.53)$ & $0.22(0.17-0.30)$ & $42(243.69)$ & $0.17(0.13-0.23)$ & $1.29(0.83-1.99)$ & $1.25(0.81-1.93)$ & -25 & 0.31 \\
\hline $\begin{array}{l}\text { Malaria diagno- } \\
\text { sis with parasitae- } \\
\text { mia }>=5000\end{array}$ & $46(241.53)$ & $0.19(0.14-0.25)$ & $31(243.69)$ & $0.12(0.09-0.18)$ & $1.48(0.92-2.38)$ & $1.42(0.88-2.30)$ & -42 & 0.15 \\
\hline Severe malaria & $3(307.2)$ & 0.01 & $6(285)$ & 0.02 & 0.45 & $0.52(0.04-7.04)$ & 48 & 0.62 \\
\hline
\end{tabular}

Table 3 Comparison of baseline and endline indices of the study

\begin{tabular}{|c|c|c|c|c|c|}
\hline Variable & Study area & Baseline & End line & $95 \% \mathrm{Cl}$ & P-value \\
\hline \multirow[t]{2}{*}{ Children with Parasitaemia, n (\%) } & Intervention & $227(31.1)$ & $82(12.1)$ & $19(14.8,23.1)$ & 0.000 \\
\hline & Non-intervention & $64(9.0)$ & $136(21.5)$ & $-12.5(-16.3,-8.6)$ & 0.000 \\
\hline \multirow[t]{2}{*}{ Children with Gametocytes, n (\%) } & Intervention & $13(1.8)$ & $22(3.2)$ & $-1.4(-3.0,-0.2)$ & 0.090 \\
\hline & Non-intervention & $4(0.6)$ & $35(5.5)$ & $-4.9(-6.7,-3.0)$ & 0.000 \\
\hline \multirow[t]{2}{*}{ Children with $\mathrm{Hb}<11.0 \mathrm{~g} / \mathrm{dl}, \mathrm{n}(\%)$} & Intervention & $386(52.8)$ & $262(38.6)$ & $14.2(9.0,19.3)$ & 0.000 \\
\hline & Non-intervention & $386(54.5)$ & $397(62.6)$ & $-8.1(-13.3,-2.8)$ & 0.003 \\
\hline \multirow[t]{4}{*}{ Children with $\mathrm{Hb}<8.0 \mathrm{~g} / \mathrm{dl}$, n (\%) } & Intervention & $26(4)$ & $11(2)$ & $2(0.2,3.7)$ & 0.028 \\
\hline & Non-intervention & $27(4)$ & $41(6)$ & $-2.0(-4.3,0.34)$ & 0.091 \\
\hline & & \multicolumn{2}{|l|}{ Baseline } & \multicolumn{2}{|l|}{ Endline } \\
\hline & & Mean & $95 \% \mathrm{Cl}$ & Mean & $95 \% \mathrm{Cl}$ \\
\hline \multirow[t]{2}{*}{ Mean Parasite Density (asexual) } & Intervention & 1883 & 1424,2489 & 3349 & 2198,5103 \\
\hline & Non-intervention & 370 & 219,626 & 1454 & 1053,2009 \\
\hline \multirow[t]{2}{*}{ Mean Parasite Density (sexual) } & Intervention & 75 & 44,127 & 209 & 133,330 \\
\hline & Non-intervention & 204 & 36,1137 & 123 & 85,180 \\
\hline \multirow[t]{2}{*}{ Total Mean Haemoglobin ( $\mathrm{Hb})$} & Intervention & 10.79 & $10.69,10.88$ & 11.18 & $11.07,11.31$ \\
\hline & Non-intervention & 10.77 & $10.66,10.88$ & 10.53 & $10.41,10.66$ \\
\hline
\end{tabular}


proportion of children carrying asexual parasites reduced significantly from $31.1 \%(95 \% \mathrm{CI} 28,36)$ to $12.1 \%$ (95\%CI $10,15)$, a difference of $19 \%(95 \% \mathrm{CI} 15,23) \mathrm{p}<0.000 .1$ (Table 3).

At baseline, the geometric mean trophozoite and gametocyte counts for intervention district were 1883 parasites/dl and 75 parasite/dl, respectively. Both increased at the end-line to 3349 parasites / $\mathrm{dl}$ and 209 parasite/dl, respectively. The number of children carrying gametocytes almost doubled (from $13(1.8 \%)$ to $22(3.2 \%)$ at the end of the SMC administration.

In the non-intervention district, the geometric mean trophozoite and gametocyte counts were 370 parasites/ $\mathrm{dl}$ and 204 parasites/dl respectively at baseline and 1452 parasites/dl and 123 parasites/dl at endline. The number of children carrying gametocytes was $4(0.6 \%)$ at baseline and 35 (5.5\%) at endline.

\section{Effect of SMC on haemoglobin (Hb) levels (Table 3 )}

The mean $\mathrm{Hb}$ level of participants at baseline in the intervention area were $10.79 \mathrm{~g} / \mathrm{dl}(95 \% \mathrm{CI} 10.69,10.88)$ and this increased to $11.18 \mathrm{~g} / \mathrm{dl}(95 \% \mathrm{CI} 11.07,11.31)$ at the end line. For the non-intervention area, the mean $\mathrm{Hb}$ levels of participants reduced from $10.77 \mathrm{~g} / \mathrm{dl}$ at baseline to $10.53 \mathrm{~g} / \mathrm{dl}$ at endline. For all participants present at the end line, there was a mean reduction in $\mathrm{Hb}$ levels of $0.24 \mathrm{~g} / \mathrm{dl}$ for participants at the non-intervention district and increase of $0.39 \mathrm{~g} / \mathrm{dl}$ for participants from the intervention district and both were significant $(\mathrm{p}<0.0001)$.

The proportion of children with $\mathrm{Hb}<11.0 \mathrm{~g} / \mathrm{dl}$ and $\mathrm{Hb}$ $<8.0 \mathrm{~g} / \mathrm{dl}$ at baseline was $52.8 \%(95 \% \mathrm{CI} 51,58)$ and $4 \%$ $(95 \%$ CI 3,5$)$ respectively in the intervention district. At the end line, the proportion of children anemic with $\mathrm{Hb}$ less than $11.0 \mathrm{~g} / \mathrm{dl}$ and $8.0 \mathrm{~g} / \mathrm{dl}$ reduced to $38.6 \%(95 \% \mathrm{CI}$ $35,43)$ and $2 \% \%(95 \% \mathrm{CI} 1,3)$ respectively and both were statistically significant $(\mathrm{P}<0.0001)$. In the non-intervention district however, both proportions increased from
54.5 to $62.6 \%$ in those with $\mathrm{Hb}<11 / 0 \mathrm{~g} / \mathrm{dl}$ and from 4 to $6 \%$ in those $\mathrm{HB}<8 \mathrm{~g} / \mathrm{dl}$ and were also statistically significant $(\mathrm{P}<0.0001)$.

\section{Adverse events experienced by participants following SMC administration (Table 4)}

Of the 731 children recruited in the intervention district, $635(87 \%)$ received the second dose, while $587(80 \%)$ received the third dose. In the last round, 621 (85\%) took the intervention drug. Most participants received the intervention except those who had travelled or had taken prior malaria treatment. There was no severe adverse event associated with the drug administration. At least $11 \%$ of children experienced one adverse event or the other during each round of administration (Fig. 4). Diarrhoea, vomiting, and fever were the most experienced adverse events during the four rounds of SMC administration. Most of the adverse events were malaria-like symptoms and were mild to moderate and all resolved within the week. The frequencies of adverse events experienced are summarized in Table 4.

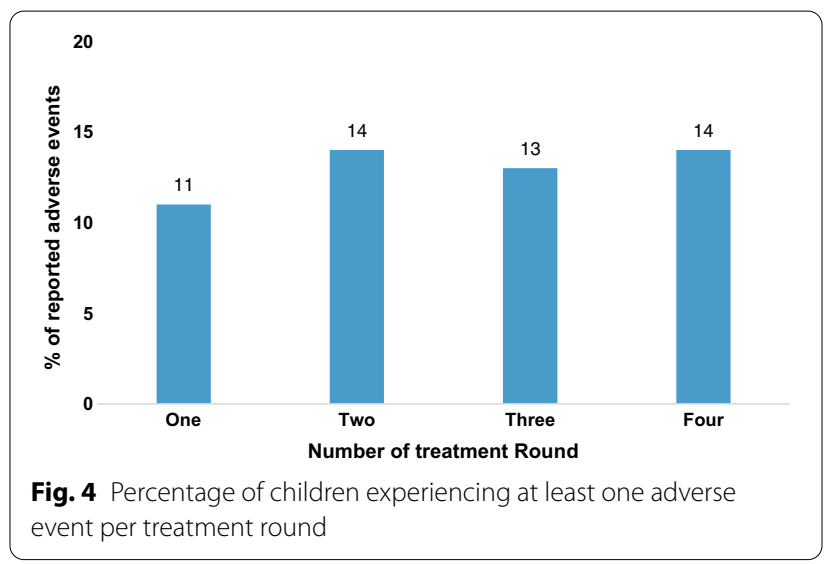

Table 4 Frequencies of Reported Adverse Event by Participants per Treatment Round

\begin{tabular}{|c|c|c|c|c|c|}
\hline \multirow[t]{2}{*}{ Adverse events } & \multicolumn{5}{|c|}{ Number per Treatment Round } \\
\hline & One $(N=731)$ & Two $(\mathrm{N}=635)$ & Three $(\mathrm{N}=587)$ & Four $(\mathrm{N}=621)$ & Tota \\
\hline Vomiting & 33 & 27 & 33 & 51 & 144 \\
\hline Fever & 7 & 19 & 32 & 27 & 85 \\
\hline Diarrhoea & 26 & 25 & 7 & 12 & 70 \\
\hline Weakness & 11 & 7 & 3 & 3 & 24 \\
\hline Abdominal pains & 0 & 5 & 0 & 0 & 5 \\
\hline Cough & 0 & 14 & 0 & 5 & 19 \\
\hline Rashes & 0 & 9 & 4 & 0 & 13 \\
\hline Others & 6 & 3 & 13 & 14 & 36 \\
\hline Total & 83 & 109 & 92 & 112 & 396 \\
\hline
\end{tabular}




\section{Discussion}

Several studies have demonstrated the efficacy of SMC under varying health systems $(3,6,7)$, however, the feasibility and potential challenges of scaling up the SMC in northern Ghana needed to be assessed under the existing public health system. The study documented how SMC as a malaria control tool could be operationally initiated and scale up in the most efficient and effective way in Ghana. This is because in Ghana, the health system depends on a complex interplay of factors including the local community dynamics, drug acceptability, climate conditions, malaria transmission dynamics and population distribution. This pilot to document the effectiveness and best practices for SMC implementation in Ghana was undertaken.

From the study results, the overall participant retention rate was very satisfactory and consistent with previous SMC studies [3]. High participant retention is important as it serves as a proxy of how effective a programme will be if it is fully scale up in normal conditions. High dropouts in programme interventions can engender staff apathy, increase implementation costs and longer duration of implementation among others. In addition, this study gave an indication on the optimal staff numbers needed for scale up and how community health volunteers could be used to implement SMC in Ghana. Parental cooperation and appreciation of the intervention are suggestive of the potential acceptance of SMC in these settings. These were further boosted by the reported adverse events which were mild and consistent with routine malaria symptoms ranging from 10 to $15 \%$.

The results also give an indication on how SMC could impact on malaria outcomes. From the results though the number of severe outcomes captured in this study was low, having six severe outcomes including death in the non-intervention compared to three in the intervention arm is an indication of potential good impact. While the difference was not statistically significant probably due to the small numbers and the sample size, as a pilot study this gives an indication of how effective the SMC intervention could be in our settings when the actual programme is implemented and scaled up. Moreover, the rate of 10 per 1000 child years at risk in the intervention arm is half that of the nonintervention group and consistent with other low rate reported elsewhere in Mali [7]. There were about $20 \%$ reduction in the proportion of children carrying parasites in the intervention areas compared to about $12 \%$ in increase in the nonintervention area. This is relevant as chronic malaria infection are known to be the major cause of anaemia in children in endemic areas. The results did not show any impact of SMC on sexual malaria parasite suggestive the drugs used may not be able to suppress transmission in the short term.

The proportion of gametocytaemia increased in both districts and the increase at the endline was much higher in the non-intervention group. This suggests that the drug combination at this present moment is not effective in killing the sexual forms of the parasite responsible for malaria transmission.

This notwithstanding, at the end of the transmission season, moderate anaemia was reduced by about $50 \%$ in the intervention arm which was consistent with earlier studies [3-8]. This was in contrast with an increased proportion of about $25 \%$ in the non-intervention area. A similar effect was documented with mild anaemia. This is noteworthy because despite the high intensity of malaria infection in the intervention area, children were protected against anaemia. Moreover, children were able to build up their haemoglobin levels as clinical and subclinical malaria parasitaemia is reduced. SMC therefore even without iron supplementation would be a good tool for the prevention of anaemia due to malaria in these children.

The safety and tolerability of SMC in the intervention area were good. There were no serious adverse events reported immediately following SMC administration and there was no report of stopping subsequent administrations due to safety of the drugs. Though severe malaria and a death were recorded these were not attributable to the drugs.

Diarrhoea and vomiting were the dominant adverse drug reactions, but they were mostly self-limiting. At least $11 \%$ of children taking the SMC medications at a time experienced adverse drug reactions and this is much higher than reported in other studies. The level of adverse drug reactions reported in this study is thus acceptable especially as they were all self-limiting and all resolved without sequelae. The study has been limited by the sample size and number of study sites.

\section{Conclusions}

The pilot study showed SMC provided substantial protection against anaemia and malaria parasite carriage in children in the Northern Sahelian belt of Ghana in addition to the other control measures like IRS and ITN use. The choice of amodiaquine and sulfadoxine-pyrimethamine is safe and tolerable among children in this region, though a drug with gametocidal effects in the future will provide additional effects in clearing gametocytes at the end of season. These findings support the proposal to scale up the implementation of SMC to the other two regions of northern Ghana. 


\section{Acknowledgements}

The authors wish to thank the study participants, their parents and the study communities' members particularly the elders and the staff and management of the Ghana Health Service of the three regions for their invaluable cooperation. We also want to thank the staff of the Navrongo Health Research Centre for their contribution to the success of the study. We are grateful to Stephen Quaye, Peter Wontuo and Stephen Appiah for the hard work which led to the success of the study.

\section{Authors' contributions}

Conceived and designed the project: POA, NAA, KM, ARO. Experimentation and data collection: POA, NAN, DA, NP, SD, YS, CM, JA, JAW, WO, ARO. Review and intellectual input: POA, NAN, KM, DA, NP, SD, YS, CM, JA, JAW, WO, ARO. All authors read and approved the final manuscript.

\section{Funding}

The project has been funded by the National Malaria Control Program with funds from the Global Fund and supported by the Ghana Government and the Navrongo Health Research Centre of the Research and Development Division of the Ghana Health Service.

\section{Availability of data and materials}

The datasets during and/or analysed during the current study available from the corresponding author on reasonable request.

\section{Declarations}

\section{Ethics approval and consent to participate}

The study was approved by the Institutional Review Board of the Navrongo Health Research Centre and the Ghana Health Service Ethics Review Committee before study implementation. All amendments were to be approved by the ethics boards before implementation, as appropriate. In addition, all participants provided parental informed consent before study enrolment and participation.

\section{Consent for publication}

All authors approved the final version of the manuscript and the corresponding author has the permission of all authors to publish this manuscript.

\section{Competing interests}

The authors declare that they have no competing interests.

\section{Author details}

${ }^{1}$ Navrongo Health Research Centre, Research and Development Division, Ghana Health Service, Navrongo, Ghana. ${ }^{2}$ Public Health Division, National Malaria Control Programme, Ghana Health Service, Accra, Ghana. ${ }^{3}$ Upper East Regional Health Directorate, Ghana Health Service, Bolgatanga, Ghana. ${ }^{4}$ Northern Regional Health Directorate, Ghana Health Service, Tamale, Ghana. ${ }^{5}$ Upper West Regional Health Directorate, Ghana Health Service, Wa, Ghana.

Received: 9 June 2021 Accepted: 7 November 2021 Published online: 18 November 2021

\section{References}

1. WHO. World Malaria Report 2020. Geneva, World Health Organization, 2020. https://www.who.int/docs/default-source/malaria/world-malariareports/9789240015791.
2. WHO. Global Technical Strategy for Malaria 2016-2030. Geneva, World Health Organization, 2015. https://www.who.int/malaria/publications/ atoz/9789241564991/en/

3. Wilson AL. A systematic review and meta-analysis of the efficacy and safety of intermittent Preventive treatment of malaria in children (IPTC). PLOS One. 2011;6:e16976.

4. WHO. Seasonal malaria chemoprevention with sulphadoxine-pyrimethamine plus amodiaquine in children: a field guide. Geneva: World Health Organization; 2013.

5. WHO. Report of the technical consultation on seasonal malaria chemoprevention (SMC Geneva, World Health Organization, 2011. http://www. who.int/malaria/publications/atoz/smc_report_teg_meetingmay2011. pdf.

6. Cairns M, Roca-Feltrer A, Garske T, Wilson A, Diallo D, Milligan P, et al. Estimating the potential public health impact of seasonal malaria chemoprevention in African children. Nat Commun. 2012;3:881.

7. Dicko A, Diallo Al, Tembine I, Dicko Y, Dara N, Sidibe Y, et al. Intermittent preventive treatment of malaria provides substantial protection against malaria in children already protected by an insecticide-treated bednet in Mali: a randomized, double-blind, placebo-controlled trial. PLoS Med. 2011:8:e1000407

8. Coldiron ME, von Seidlein L, Grais RF. Seasonal malaria chemoprevention: successes and missed opportunities. Malar J. 2017;16:481.

9. Sokhna C, Cisse B, Hadj Bâ E, Milligan P, Hallett R, Sutherland C, et al. A trial of the efficacy, safety and impact on drug resistance of four drug regimens for seasonal intermittent preventive treatment in Senegalese children. PLoS One. 2008;3:e1471.

10. Onyango EO, Ayodo G, Watsierah CA, Were T, Okumu W, Anyona SB, et al. Factors associated with non-adherence to artemisinin-based combination therapy (ACT) to malaria in a rural population from holoendemic region of western Kenya. BMC Infect Dis. 2012;12:143.

11. Annual Situational Report 2014. National Malaria Control Programme (NMCP), Ghana Health Service. Ministry of Health Ghana.

12. Oduro AR, Wak G, Azongo D, Debpuur C, Wontuo P, Kondayire F, et al. Profile of the Navrongo Health and Demographic Surveillance System. Int J Epidemiol. 2012;41:968-76.

13. Ghana Statistical Service (2014). District Analytical Report. Lawra district

14. Ghana Statistical Service (2014). District Analytical Report. West Mamprusi district

15. Koram KA, Owusu-Agyei S, Fryauff DJ, Anto F, Atuguba F, Hodgson A, et al. Seasonal profiles of malaria infection, anaemia, and bednet use among age groups and communities in northern Ghana. Trop Med Int Health. 2003;8:793-802.

16. Oduro AR, Koram KA, Rogers W, Atuguba F, Ansah P, Anyorigiya T, et al. Severe falciparum malaria in young children of the Kassena-Nankana district of northern Ghana. Malar J. 2007:6:96.

17. Appawu M, Owusu-Agyei S, Dadzie S, Asoala V, Anto F, Koram K, et al. Malaria transmission dynamics at a site in northern Ghana proposed for testing malaria vaccines. Trop Med Int Health. 2004;9:164-70.

18. Nyonator FK, Awoonor-Williams JK, Phillips JF, Jones TC, Miller RA. The Ghana community-based health planning and services initiative for scaling up service delivery innovation. Health Policy Plan. 2005;20:25-34.

\section{Publisher's Note}

Springer Nature remains neutral with regard to jurisdictional claims in published maps and institutional affiliations. 